\title{
Measurement of lingual vibrotactile sensitivity using pulsed and continuous stimulation*
}

\author{
DONALD J. FUCCI and DENNIS J. ARNST \\ Hearing and Speech Sciences \\ Ohio University, Athens, Ohio 45701
}

\author{
KAL M. TELAGE \\ State University of New York \\ Geneseo, N.Y. 14454
}

and

\author{
PATRICK McCAFFREY \\ Hearing and Speech Sciences \\ Ohio Univeristy, Athens, Ohio 45701
}

\begin{abstract}
Vibrotactile thresholds were obtained from the anterior midline surface of the tongue in four groups of 50 normal-speaking young adults. Thresholds were measured using an ascending-pulsed, ascending-continuous, descending-pulsed, and descending-continuous stimulus series. Statistical analyses indicated that thresholds obtained with the descending-continuous stimuli were least sensitive. This effect on vibrotactile thresholds appeared to be related to perstimulatory fatigue much like the same phenomenon reported with obtaining auditory thresholds.
\end{abstract}

Tactile sensitivity in the oral area has been used to assess normal function of sensory receptors contributing to the closed-loop feedback system active during speech. The oral tactile sensory system has previously been established as an important aspect of the speech monitoring mechanism (Van Riper \& Irwin, 1962; Mysak, 1966). Continuous closed-loop tracking of speech sounds is not only reflected in the sound patterns of speech, but also related to specific somesthetic processes associated with normal articulatory movements (Smith \& Henry, 1967).

Fucci (1972) reported differences between normal speaking and "functional" speech-defective adults when comparing vibrotactile thresholds obtained from the lip and tongue. Speech-defective Ss were found to be less sensitive for oral vibrotactile stimulation than unaffected speakers. The implication of this work suggested that poor oral vibrotactile sensitivity reflects an interruption in the sensory feedback mechanism utilized for proper control of the articulators.

Since vibration has been shown to be a successful means of assessing peripheral and central tactile processes (Geldard, 1940), application of vibrotactile stimulation to the oral area appears to provide an adequate measure of tactile feedback mechanisms utilized for speech.

Various phychophysical methods have been evaluated in terms of effects on vibrotactile threshold results. Hall,

*This paper is sponsored by James L. Bruning, who takes full editorial responsibility for its contents.
Fucci, \& Arnst (1972) found no differences between data obtained with either the method of limits or the method of adjustment. Differences between ascending and descending thresholds obtained with pulsed stimulation were also found to be insignificant. However, an ascending procedure was recommended for use in threshold testing to avoid any possible adaptation effects.

Fucci, Arnst, \& Telage (1972) reported that thresholds obtained with a descending-continuous stimulus produced significantly less sensitive thresholds than either ascending-pulsed or ascending-continuous procedures. These results were similar to those reported in Hirsh (1952) for auditory threshold data. The fact that continuous thresholds were less sensitive when using a descending series suggested the presence of some adaptation.

In an attempt to define appropriate vibrotactile threshold procedures, the present study was designed to compare ascending and descending thresholds using pulsed and continuous stimulation. The four conditions under consideration in this study were ascending-pulsed, ascending-continuous, descending-pulsed, and descending-continuous.

\section{METHOD}

Ss in the present study ranged in age from 18 to 36 years and reported no history of speech, sensory, and/or motor impairments. Lingual vibrotactile thresholds were obtained on 200 Ss, using ascending-pulsed, ascending-continuous, descending-pulsed, and descending-continuous stimulation. Fifty different Ss were evaluated under each condition.

The equipment used in this study has been described in a previous publication (Telage, Fucci, \& Arnst, 1972). The stimulus unit included a sine-wave generator, frequency counter, electronic switch (designed to maintain a sine-wave configuration), interval timer, amplifier, variable attenuator, and electromagnetic vibrator. The pulsed vibratory signal generated had a 50\% duty cycle (on $1 \mathrm{sec}$ and off $1 \mathrm{sec}$ ) with a rise and decay time of $100 \mathrm{msec}$. The measurement unit consisted of an accelerometer, cathode follower, microphone amplifier, and vacuum tube voltmeter. A white-noise generator was used to present auditory masking at $100-110 \mathrm{~dB}$ SPL to Ss through TDH-39 headphones.

Each S was seated in a dental chair and extended his tongue between two sterilized plastic disks which clamped the structure in place tightly enough to hold it without affecting normal circulation. The stimulus was applied to the dorsal anterior midline surface of the tongue through a free surround disk containing a hole with a diameter $2 \mathrm{~mm}$ greater than that of the contactor. The contactor area was $.128 \mathrm{~cm}^{2}($ diam $=.404 \mathrm{~cm})$ and was within a range reported by Verrillo (1963).

The vibrator was mounted on an adjustable drill press platen and could be lowered into position. In order to maintian constant pressure of the vibrator. on the tongue of all Ss. the unit was lowered $1 \mathrm{~mm}$ beyond initial contact with the tongue. This was accomplished by using a simple circuit consisting of an ohmmeter with its negative wire connected to the base of the 
Table 1

Means Obtained for Each Group of Data

\begin{tabular}{lc}
\hline \multicolumn{1}{c}{$\begin{array}{c}\text { Threshold } \\
\text { Data }\end{array}$} & $\begin{array}{c}\text { Mean } \\
\text { (in microns) }\end{array}$ \\
\hline Ascending-Pulsed & .180 \\
Ascending-Continuous & .184 \\
Descending-Pulsed & .380 \\
Descending-Continuous & .567 \\
\hline
\end{tabular}

electromagnetic vibrator and its positive wire between the S's thumb and forefinger.

Lingual vibrotactile thresholds were obtained at $300 \mathrm{~Hz}$ for all Ss by using a method of limits with either an ascending-pulsed, ascending-continuous, descending-pulsed, or descending-continuous series of stimulation. The $\mathrm{S}$ was required to respond by raising his hand as soon as he either detected or ceased to detect the stimulus under the appropriate condition. A median of $3-\mathrm{mV}$ readings was accepted as the lingual threshold and was later changed to displacement in microns with a standard $\mathrm{g}$ formula for acceleration conversion.

\section{RESULTS AND DISCUSSION}

Data in microns were averaged across Ss for the ascending-pulsed, ascending-continuous, descending-pulsed, and descending-continuous conditions. These results appear in Table 1. Both descending conditions produced less sensitive thresholds than either of the ascending conditions. Moreover, the descending-continuous threshold appears to be the least sensitive when compared with the other three conditions. In order to determine significant differences in the data, thresholds in microns of displacement were submitted to a two-factor analysis of variance with the alpha level set at .01 . It can be seen from Table 2 that both main effects and their interaction produced significant $\mathrm{F}$ ratios.

A Tukey multiple comparisons test was used to detect significant differences between the individual means for each condition. Based on this analysis, only the ascending-pulsed and ascending-continuous thresholds did not differ significantly. Ascending-pulsed and

Table 2

Summary Table for the Analysis of Variance

\begin{tabular}{lrrr}
\hline \multicolumn{1}{c}{ Source } & df & MS & \multicolumn{1}{c}{ F } \\
\hline Psychophysical Method (A) & 1 & 0.459 & $35.410^{*}$ \\
Stimulus Pattern (B) & 1 & 4.252 & $328.073^{*}$ \\
A by B & 1 & 0.421 & $32.515^{*}$ \\
Error & 196 & 0.013 & \\
\hline
\end{tabular}

${ }^{*} p<.01$ ascending-continuous thresholds were significantly more sensitive than either descending threshold conditions. However, descending pulsed data were more sensitive than descending continuous.

It appears from the data that descending-continuous stimulation produces the greatest effect on vibrotactile thresholds obtained from the tongue. Furthermore. descending-pulsed thresholds were also shown to be significantly different from either of the ascending conditions. The poorer thresholds obtained when using a descending technique may be suggestive of the same type of perstimulatory fatigue factor that has been identified with auditory thresholds. In this case, thresholds obtained with a descending-continuous tone are affected by neural fatigue, causing the system to be less responsive to the stimulus.

It can be concluded that vibrotactile thresholds obtained from the anterior midline portion of the tongue are affected by the type of stimulation used. This finding further supports the recommendation of Hall, Fucci, \& Arnst (1972) to use an ascending stimulus series for vibrotactile studies on the tongue. It also provides some basis for exploring suprathreshold fatigue factors and their effect on tactile processing utilized during the speech act.

\section{REFERENCES}

Fucci, D. J Oral vibrotactile sensation: An evaluation of normal and defective speakers. Journal of Speech \& Hearing Research, 1972, 15, 179-184.

Fucci, D., Arnst, D., \& Telage, K. The effects of pulsed and continuous stimulation on vibrotactile thresholds obtained from the tongue. Psychonomic Science, 1972, 29, 83-84.

Geldard, F. A. The perception of mechanical vibration: IV. Is there a separate vibratory sense? Journal of General Psychology, 1940, 22, 291-308.

Hall, D. E., Fucci, D. J., \& A rnst, D. J. Vibrotactile stimulation: An investigation of psychological methods for establishing threshold. Perceptual \& Motor Skills, 1972, 34, 891-898.

Hirsh, I. J. The measurement of hearing. New York: McGraw-Hill, 1952.

Mysak, E. D. Speech pathology and feedback theory. Springfield, Ill: Thomas, 1966.

Smith, K. U., \& Henry, J. P. Cybernetic foundations for rehabilitation. American Journal of Physical Medicine, 1967. 46, 379-467.

Telage, K., Fucci, D., \& Arnst, D. Normative study of oral vibrotactile sensitivity. Journal of Perceptual \& Motor Skills, 1972, 35, 671-676.

Van Riper, C., \& Irwin, J. Voice and articulation. Englewood Cliffs: Prentice-Hall, 1962.

Verrillo, R. T. Effect of contactor area on the vibrotactile threshold. Journal of the Acoustical Society of America, $1963,35,1962-1966$.

(Received for publication May 28, 1973.) 